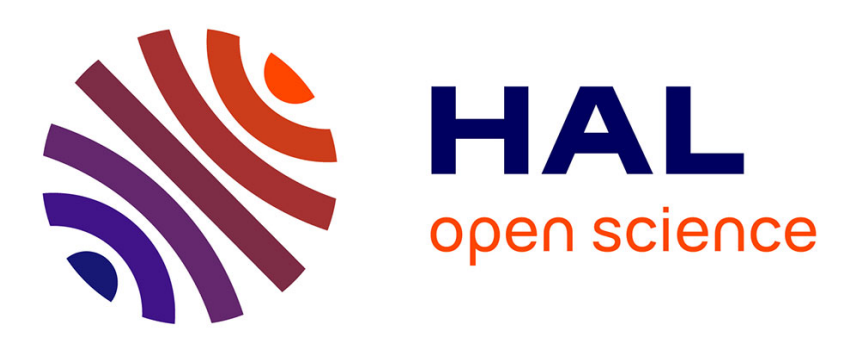

\title{
Far-Field Radiation of Three-Dimensional Plasmonic Gold Tapers near Apexes
}

Surong Guo, Nahid Talebi, Alfredo Campos, Wilfried Sigle, Martin Esmann, Simon F Becker, Christoph Lienau, Mathieu K Kociak, Peter A van Aken

\section{- To cite this version:}

Surong Guo, Nahid Talebi, Alfredo Campos, Wilfried Sigle, Martin Esmann, et al.. Far-Field Radiation of Three-Dimensional Plasmonic Gold Tapers near Apexes. ACS photonics, 2019, 6 (10), pp.2509-2516. 10.1021/acsphotonics.9b00838 . hal-02352227

\section{HAL Id: hal-02352227 \\ https://hal.science/hal-02352227}

Submitted on 6 Nov 2019

HAL is a multi-disciplinary open access archive for the deposit and dissemination of scientific research documents, whether they are published or not. The documents may come from teaching and research institutions in France or abroad, or from public or private research centers.
L'archive ouverte pluridisciplinaire HAL, est destinée au dépôt et à la diffusion de documents scientifiques de niveau recherche, publiés ou non, émanant des établissements d'enseignement et de recherche français ou étrangers, des laboratoires publics ou privés. 


\title{
Far-Field Radiation of Three-Dimensional Plasmonic Gold Tapers near Apexes
}

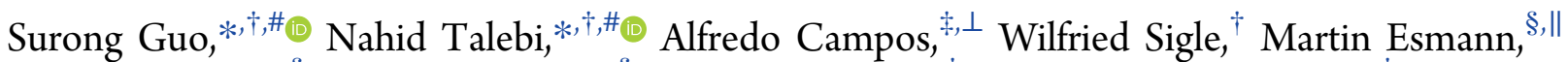 \\ Simon F. Becker, ${ }^{\S}$ Christoph Lienau, ${ }^{\S}$ Mathieu Kociak, ${ }^{\ddagger}{ }^{\ddagger}$ and Peter A. van Aken ${ }^{\dagger}$ \\ ${ }^{\dagger}$ Max Planck Institute for Solid State Research, Heisenbergstraße 1, 70569 Stuttgart, Germany \\ ${ }^{\ddagger}$ Laboratoire de Physique des Solides, Université Paris-Sud, Orsay 91400, France \\ ${ }^{\S}$ Institute of Physics and Center of Interface Science, Carl von Ossietzky University Oldenburg, 26129 Oldenburg, Germany
}

Supporting Information

\begin{abstract}
Three-dimensional plasmonic gold tapers are widely used structures in nano-optics for achieving imaging at the nanometer scale, enhanced spectroscopy, confined light sources, and ultrafast photoelectron emission. To understand their radiation properties further, especially in the proximity of the apex at the nanoscale, we employ cathodoluminescence spectroscopy with high spatial and energy resolution. The plasmon-induced radiation in the visible spectral range from three-dimensional gold tapers with opening angles of $13^{\circ}$ and $47^{\circ}$ is investigated under local electron excitation.
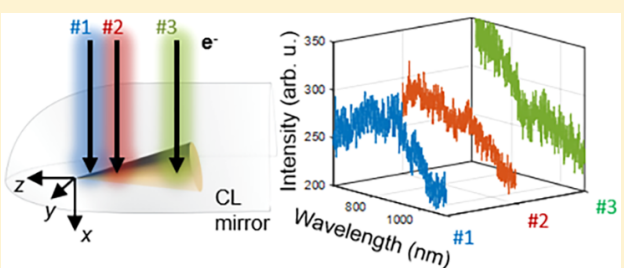

We observe a much weaker radiation from the apex of the $13^{\circ}$ taper than from that of the $47^{\circ}$ taper. By means of finitedifference time-domain simulations we show that for small opening angles plasmon modes that are created at the apex are efficiently guided along the taper shaft. In contrast for tapers with larger opening angles, generated plasmon polaritons experience larger radiation damping. Interestingly, we find for both tapers that the most intense radiation comes from locations a few hundreds of nanometers behind the apexes, instead of exactly at the apexes. Our findings provide useful details for the design of plasmonic gold tapers as confined light sources or light absorbers.
\end{abstract}

KEYWORDS: cathodoluminescence, plasmonics, surface plasmon coupled emission, gold taper

$\mathrm{T}$ hree-dimensional plasmonic gold tapers have stimulated broad interest in nano-optics due to their capability of focusing light at the subwavelength regime, in a coherent, broadband, and controllable scheme. ${ }^{1,2}$ This kind of structure is often implemented in near-field microscopes as a nanoantenna for enhancing and collecting detectable signals ${ }^{3-5}$ or in point-projection electron microscopes for providing ultrafast photoelectron emission. $^{6-12}$ Basically, the optically induced polarization of the nanostructures can be efficiently coupled to the fundamental plasmonic modes of the gold tapers and propagate along their shafts and/or radiate toward the detector. Such coupling effects are mediated by plasmons localized at the sharp apexes of these gold tapers. Thus, surface plasmons are bound to the taper shafts that essentially enable the nanofocusing of electromagnetic fields. In such azimuthally symmetric structures, there are different azimuthal orders of the sustained surface plasmons. ${ }^{13,14}$ Their behaviors are sensitive to the local radii of tapers. ${ }^{15,16}$ The fundamental mode with mode order $m=0$ has a rotationally symmetric electric field. It bonds evanescently to the surface at both larger and smaller local radii and can be eventually focused to the taper apex, ideally, for arbitrarily sharp pointed tapers, in an adiabatic nonradiating scheme. ${ }^{17,18}$ For realistic taper geometries, both radiation and reflection of $m=0$ at the apex, however, have been observed. ${ }^{6,19}$ In contrast, other plasmon modes with higher azimuthal orders $(|m|>0)$ are supposed to be excited and propagate evanescently only above specific critical radii. ${ }^{13,16}$ They radiate off the taper at a finite distance from the apex. ${ }^{20}$ With such diverse behaviors of the sustained surface plasmons on plasmonic tapers, one can readily recognize the complexity of quantifying the coupling efficiency of the excitation of each individual surface plasmon mode, which are eventually reflected from apexes, coupled to the farfield, or damped as internal losses in the system. Moreover, the performance could be mutually affected by a few variables, e.g., excitation scheme, ${ }^{21}$ opening angles of tapers, ${ }^{22}$ the curvature at the very apex of these tips, ${ }^{15}$ and surface quality.

Two nano-optical characterization techniques, electron energy-loss spectroscopy (EELS) and cathodoluminescence spectroscopy (CL), in scanning transmission electron microscopy (STEM) provide access to the local optical response of objects at the nanometer scale $\mathrm{e}^{23-26}$ and hence offer a possibility to answer the above-mentioned questions. In both techniques, fast electrons are used as a probe, which can easily excite surface plasmons via their evanescent electromagnetic field by matching momentum and energy. While EELS maps the photonic local density of states through a primary excitation process, CL probes the radiative damping during the relaxation procedure. ${ }^{23,27-29}$ Furthermore, their contrast-

Received: June 10, 2019

Published: September 17, 2019 


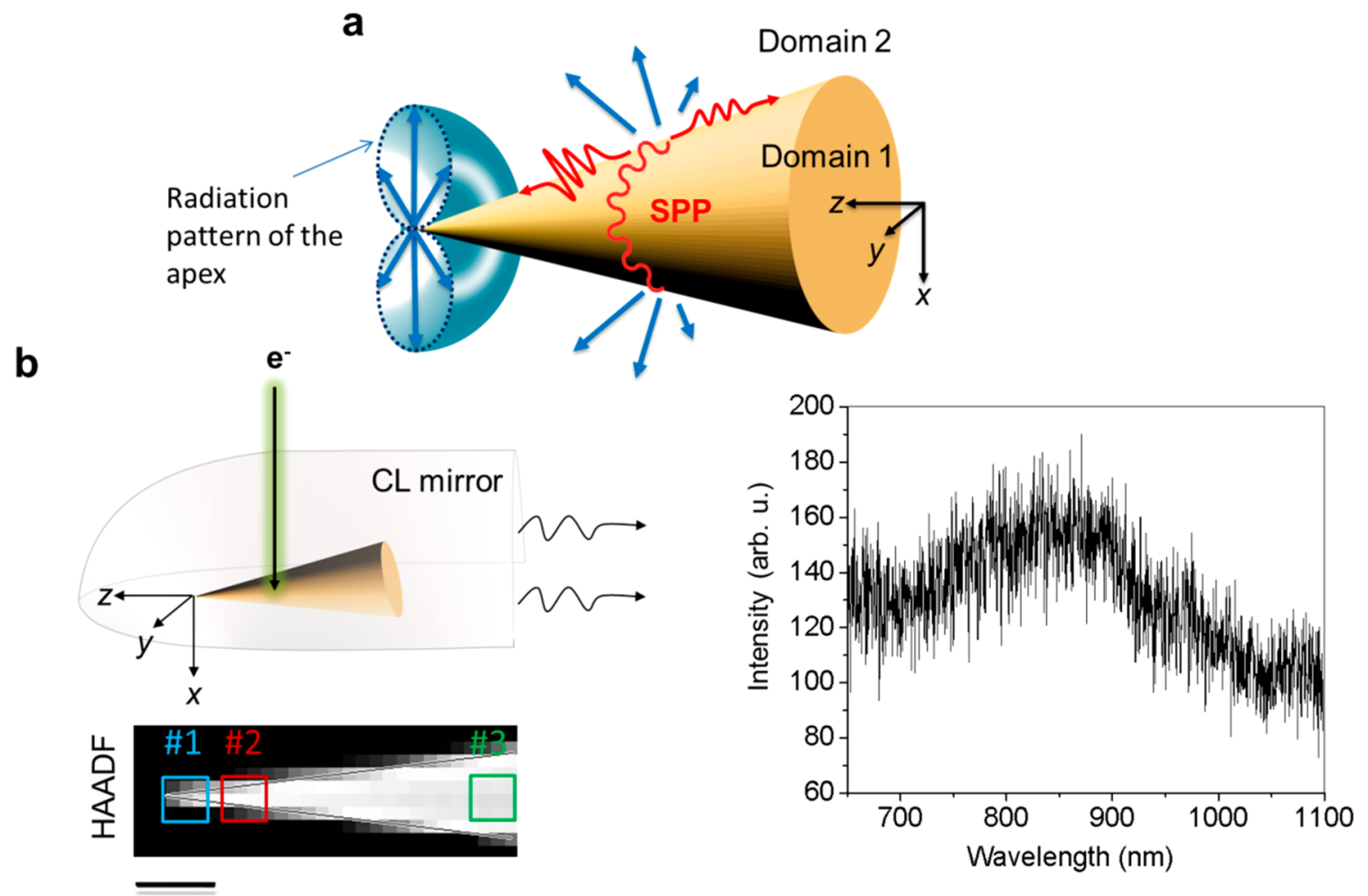

\section{C}

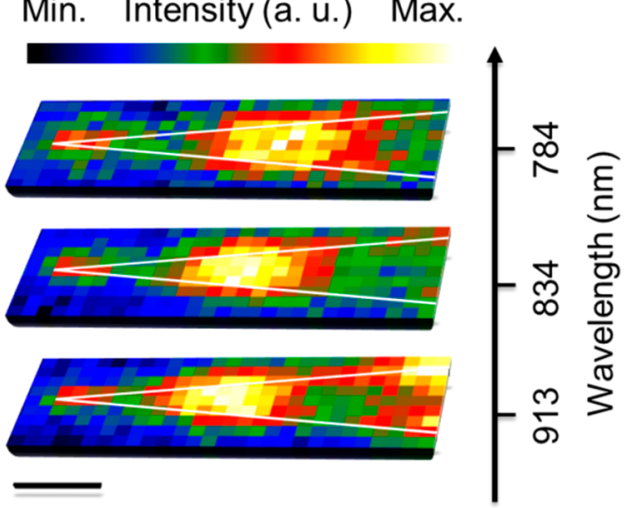

d

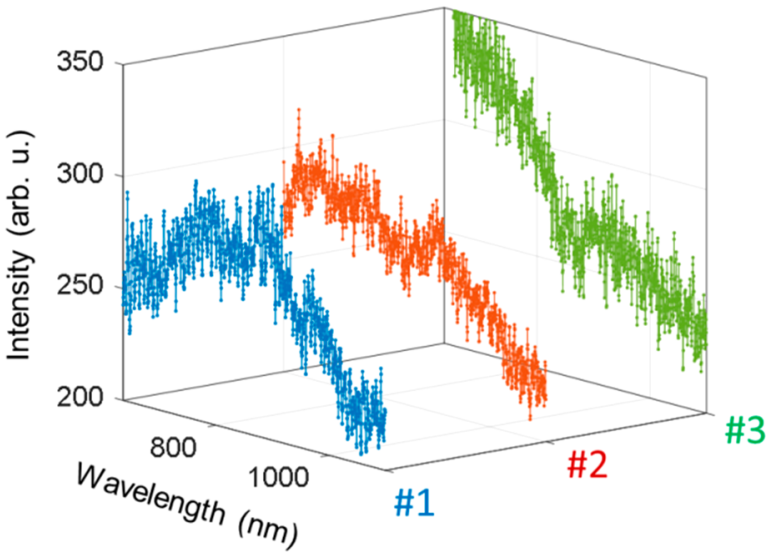

Figure 1. (a) Schematic illustration of surface-plasmon radiative decay in gold tapers. Red curves indicate the propagation of SPPs in forward, backward, and azimuthal directions. Blue lines depict the light emission due to the radiative decay of SPPs. (b) Sketches for the collection of a CL spectrum using a parabolic mirror. The taper is oriented along the optical axis of the mirror. A high-angle annular dark-field image of an investigated gold taper is inserted below. (c) Two-dimensional photon maps at selected wavelengths in CL experiments of an investigated taper. The scale bars in (b) and (c) are $100 \mathrm{~nm}$. (d) Representative CL spectra recorded at different positions on the taper, marked by colored boxes in (b).

ing juxtaposition presents nonradiative losses, e.g., heating and inter/intraband transitions. The combined study using both EELS and CL on identical objects has demonstrated interesting physical insights into the investigated nanoobjects. $^{30-33}$

For gold tapers, EELS has been applied to investigate the supported plasmonic modes in details as well as their interaction mechanism with fast electrons. ${ }^{13,19}$ It has been noticed that the opening angle of tapers plays a huge role in manipulating the formation of the supported surface plasmons $(|m|>0)$ and the field enhancement at apexes. ${ }^{22,34}$

In the present study, we employed CL to investigate the influence of the opening angle on optical far-field radiation from the proximity of the gold taper apex as a complement to our previous EELS investigations. ${ }^{13}$ Two tapers with different opening angles $\left(13^{\circ}\right.$ and $\left.47^{\circ}\right)$ were surveyed. A diverse radiating behavior is observed between these two tapers at their apexes. Corresponding finite-difference time-domain (FDTD) numerical simulations reveal the underlying reason for this experimental observation: the excited surface plasmons are either guided away from the apex along the taper or couple to the far-field radiation depending on the tapers' opening angle.

\section{RESULTS AND DISCUSSION}

The surface-plasmon-induced radiation of plasmonic tapers is schematically illustrated in Figure 1a. It can be generally catalogued as the radiation from the $m=0$ mode and higher 


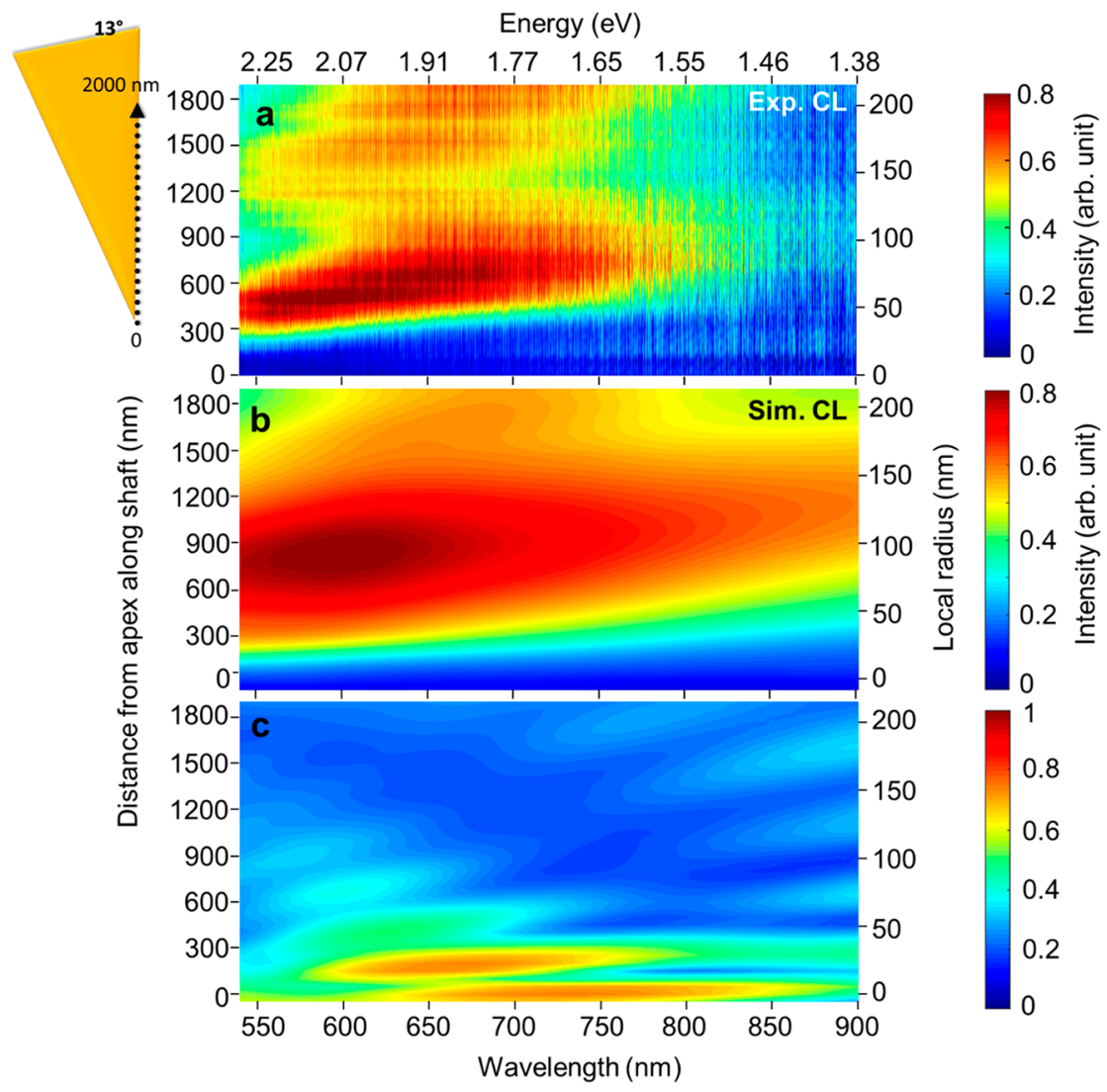

Figure 2. (a) Experimental and (b) simulated CL spectra of a taper with a $13^{\circ}$ opening angle against the distance from the apex along its shaft (left) and the local radius (right). The inset on the upper left indicates the scanning direction of the electron beam with respect to the taper. (c) Ratio of the energy guided in the $-z$ direction to the total energy collected along all orthogonal directions, taken from the simulations in (b).

order modes $(|m|>0)$. In more detail, the wave function of the taper's optical modes (which are assigned to the electric and magnetic vector potentials $\left.{ }^{13}\right)$, in analogy to optical modes in fibers, might be constructed as $\psi^{1,2}(x, y, z, t)=\sum_{m} \alpha_{m} B_{m}\left(k_{\rho}^{1,2} \rho\right) \exp (i m \varphi) \exp \left(i k_{z} z\right)$, where $(x, y)=\rho(\cos (\phi), \sin (\phi))$ and $z$ is along the rotational axis of the taper. $\alpha_{m}$ is the amplitude function for each order $m . B_{m}=$ $J_{m}$ is the Bessel function of order $m$, for fields inside the fiber (domain 1), and $B_{m}=H_{m}{ }^{(1)}$ is the Hankel function of first kind and order $m$, for fields outside the taper (domain 2, see Figure 1a). Moreover, for the domains 1 and $2,\left(k_{\rho}{ }^{1}\right)^{2}+k_{z}{ }^{2}=\varepsilon_{r} k_{0}^{2}$ and $\left(k_{\rho}^{2}\right)^{2}+k_{z}^{2}=k_{0}^{2}$, respectively, where $k_{0}$ is the wavenumber of light in free space. The fundamental mode of a metallic conical taper is then understood as the rotationally symmetric mode with $m=0$ and with the only nonzero field components given by $E_{\rho}, E_{z}$ and $H_{\phi}$. As mentioned, plasmonic gold tapers act as mode filters such that only the $m=0$ mode can propagate until the geometric singularity of the apex. It then partially radiates, with a radiation pattern similar to that of a dipole orientated along the taper axis (Figure 1a). Meanwhile, the modes with $|m|>0$ mainly radiate in transverse directions, e.g., the $x y$ plane (see Figure S4 in the Supporting Information). Therefore, the luminescence caused by surface-plasmon decay is generally suppressed in the $z$ direction, i.e., along the taper axis. For these reasons we have carefully positioned the taper parallel to the mirror axis, in order to detect the radiation from the apex as well as higher order modes. In CL experiments, a focused electron beam raster scans a region of interest in the specimen covering $N \times$
$M$ pixels in the $y z$ plane. At each pixel, the emitted photons from the entire structure impinging on the parabolic CL mirror are directed toward an external spectrometer to generate a spectrum, plotting the signal intensity as a function of emitted wavelength (Figure $1 \mathrm{~b}$ ). Geometrically speaking, the radiation in the $-x,+y,-y$, and $+z$ directions for the $x \leq 0$ domain can be well detected with the experimental setup, which assembles a great portion of the radiation from surface plasmons in the investigated structure. At the end of the scan, a threedimensional data set of hyperspectral images is obtained. Different representations of this data set may be chosen to display the information contained in this data set. For example, at specific wavelengths, two-dimensional monochromatic photon maps can be selectively visualized to reveal the spatial distribution of the detected radiation (Figure 1c), or a series of spectra can be extracted along the taper shafts for studying the influence of local variables on the spectral features (Figure 1d).

With the above knowledge, we first analyze the results for a taper with an opening angle of $13^{\circ}$. Figure $2 \mathrm{a}$ shows the experimental CL intensity as a function of the emitted wavelength and the distance from the apex along the shaft. As the tip has a constant angle and is mounted with its taper axis pointing along the optical axis of the parabolic mirror, the distance from the apex corresponds to a well-defined local radius of the taper, also shown as an extra axis on Figure 2. In the proximity of the taper apex, there is only a very faint radiation detected at the wavelengths spanning from 700 to $1000 \mathrm{~nm}$. Further away from the apex, a strong emission in the wavelength range of 500-700 $\mathrm{nm}$ rises up. Moreover, this radiation is red-shifted when the impact distance increases 


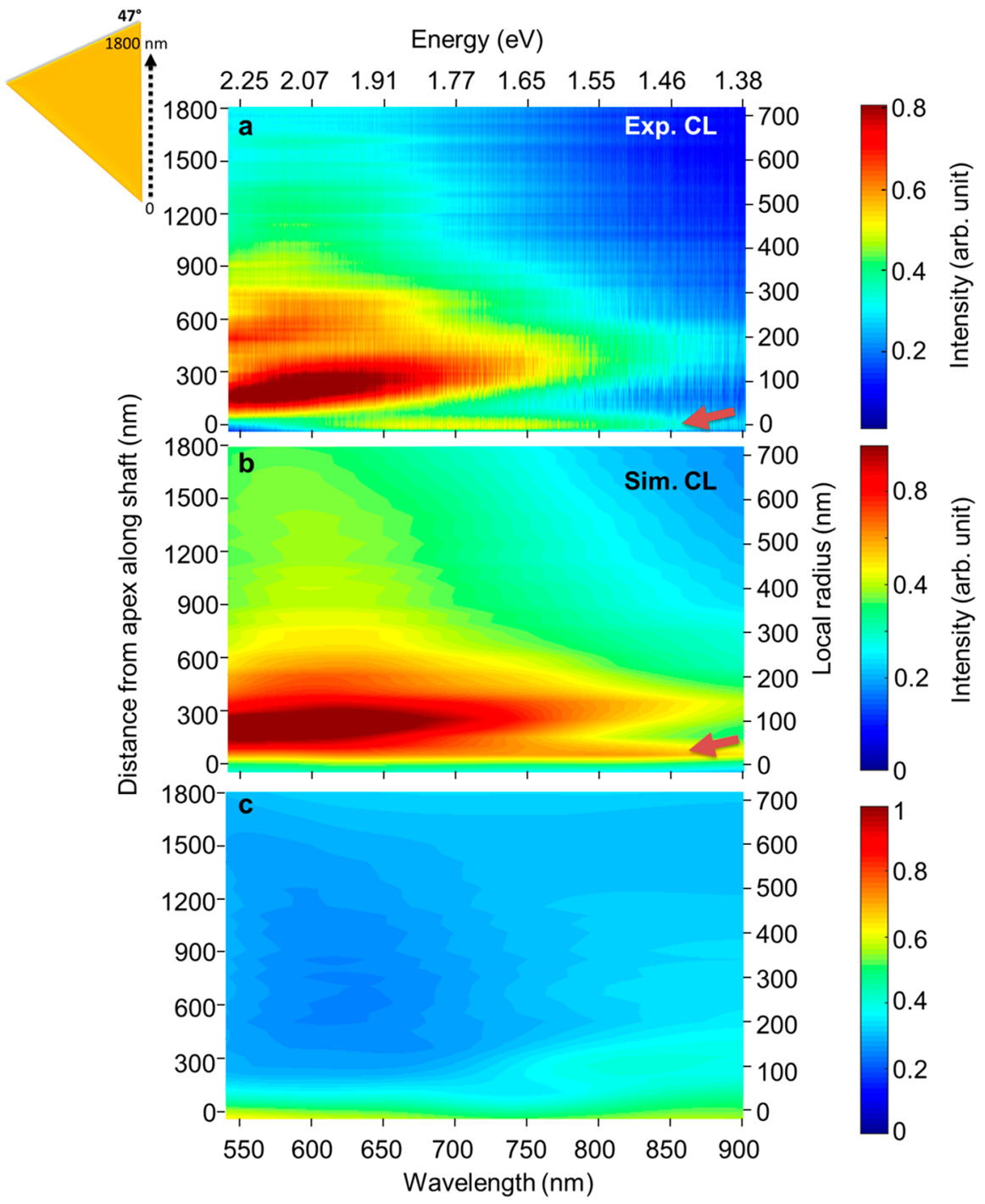

Figure 3. (a) Experimental and (b) simulated CL spectra of a gold taper with $47^{\circ}$ opening angle as a function of distance from the apex along the shaft. The inset on the left indicates the scanning direction of the electron beam with respect to the taper. Red arrows indicate the broad emission at the apex. (c) Ratio of the energy guided in the $-z$ direction to the total energy collected along all orthogonal directions.

from $300 \mathrm{~nm}$ to $800 \mathrm{~nm}$ approximately and then becomes attenuated at larger distances. It is hard to distinguish different mechanisms of radiation directly from the recorded spectra, since there are no significant differences in the spectral responses.

The corresponding simulated CL spectra are presented in Figure $2 \mathrm{~b}$. These spectra were obtained by integrating the Poynting vectors of the electron-induced radiation, obtained by numerically solving the full Maxwell's equations, across planes normal to the $-x,+y,-y$, and $+z$ directions in the $x \leq 0$ half-space (for details see the Methods section).

No significant radiation at the apex and a major radiation with maxima around 500-700 $\mathrm{nm}$ along the shaft demonstrate a high similarity to the experimental CL spectra, although we notice that the major radiation appears around $800 \mathrm{~nm}$ away from the apex, showing a distance discrepancy compared to the experimental value. This might be due to the real geometry of the taper, which differs slightly from the ideal mathematical conical shape considered in the simulations. In addition, it may be partly accounted for by the unequal and directiondependent reflectance of the CL mirror, which has not been considered in the simulations. The good agreement between the experimental and simulated CL spectra implies that in general far-field interference effects caused by coherent radiation from individual transverse electric and transverse magnetic modes are the prime phenomena for the gold tapers' cathodoluminescence. Nevertheless, further simulations also confirm that the radiative decay due to inter/intra-band transitions has negligible contributions to the luminescence of the gold taper (see Figures S1 and S2 in the Supporting Information).

At first sight, one may intuitively expect the taper apex as a dominant nanoconfined source of far-field radiation. ${ }^{36-39}$ However, in a reciprocal picture to adiabatic nanofocusing in plasmonic tapers, the $m=0$ mode is exclusively excited at the apex and is able to propagate evanescently away from apexes along the shaft. Therefore, we computed the power being guided away from apex along the shaft at a distance of $5 \mu \mathrm{m}$, which was represented by the $-z$ component of the Poynting vector based on the above-mentioned numerical simulations. Figure $2 \mathrm{c}$ shows the reflected power normalized to the summation of powers radiated along all six orthogonal 
directions as a function of distance and local radius. Very interestingly, the plot shows that as much as $75 \%$ of the electron-induced energy is guided along the $z$ direction within a distance of $400 \mathrm{~nm}$ from the apex (corresponding to local radii below $50 \mathrm{~nm}$ ). Exactly in this radius regime, the highly guided $m=0$ mode is mainly excited. ${ }^{13}$ The above results clearly show that the back-propagation of the excited surface plasmons is attributed to the weak radiation from the taper apex.

In addition, the two maxima of the guided power below a radius of $50 \mathrm{~nm}$ correspond to the excitation of the $m=0$ mode at the apex and the location for forming the first-order resonance due to the constructive interference between the forward propagating $m=0$ mode and its reflection from the apex (Figure 2c). Interferences between the forward propagating $m=0$ and its reflection from the apex may give rise to distance-dependent intensity variations in the $C L$ spectra, ${ }^{19}$ which are more clearly seen in EELS spectra of tapers with smaller opening angles. ${ }^{34}$ The major radiation between 500 and $700 \mathrm{~nm}$ behind the apex is mainly due to the radiation decay of the $m=1$ mode, as discussed in Supplementary Figure S3. In addition to the taper with a small opening angle, we also performed CL experiments on a taper with a larger opening angle of $47^{\circ}$. The measured CL spectra along the taper shaft are shown in Figure 3a. Now, strong radiation at the apex of the taper with a $47^{\circ}$ opening angle is clearly observed in the range of 600 to $800 \mathrm{~nm}$, in stark contrast to that for the $13^{\circ}$ taper's spectra (indicated by the red arrow in Figure 3a). When moving away from the apex, predominant light emission in the spectral range between 540 and $800 \mathrm{~nm}$ is detected, which is similar to the spectra of the $13^{\circ}$ taper. In the simulated CL spectra, both the clear radiation at the apex and the dominant emission along the shaft are present, displaying a very good agreement with the experimental results (Figure $3 \mathrm{~b}$ ).

Furthermore, the guided power along the $-z$ direction is generally much weaker for the $47^{\circ}$ taper shaft, except for the region close to the very apex of the tip (Figure 3c). This significant difference from the $13^{\circ}$ taper is most likely due to the drastic change of the local radius, which is 3.5 times larger than the $13^{\circ}$ taper. Therefore, adiabatic conditions for mode propagation toward the $-z$ direction mostly fail, leading to a strong coupling to the radiation of the excited mode at the taper apex. Similar to the major radiation in the $13^{\circ}$ taper, the dominant emission along the $47^{\circ}$ taper shaft mainly occurs by the decay of the modes $|m| \geq 1$.

As obviously seen from the CL spectra, the most intense radiation is not exactly emitted from the very apexes of both tapers. We have integrated the intensity of the experimental CL spectra from wavelength 540 to $900 \mathrm{~nm}$ along each taper shaft to find out where the maximum radiation is collected (Figure 4). The corresponding maximum for the $13^{\circ}$ taper appears at around $500 \mathrm{~nm}$ behind the apex along the shaft (black arrow in the blue spectrum) and for the $47^{\circ}$ taper at around a distance of $300 \mathrm{~nm}$ from the apex (black arrow in the red spectrum). In this regime the excitation of higher order plasmonic modes with $m \geq 2$ is weak since these modes are not bound to the surface. ${ }^{16}$ Due to its strong field confinement, the bound $m=0$ mode can only be excited by focusing the electron beam to the apex region of the taper and thus also does not contribute significantly to the strong radiation that is seen when exciting away from the apex. Therefore, we correlated these radiation maxima mainly with the radiative
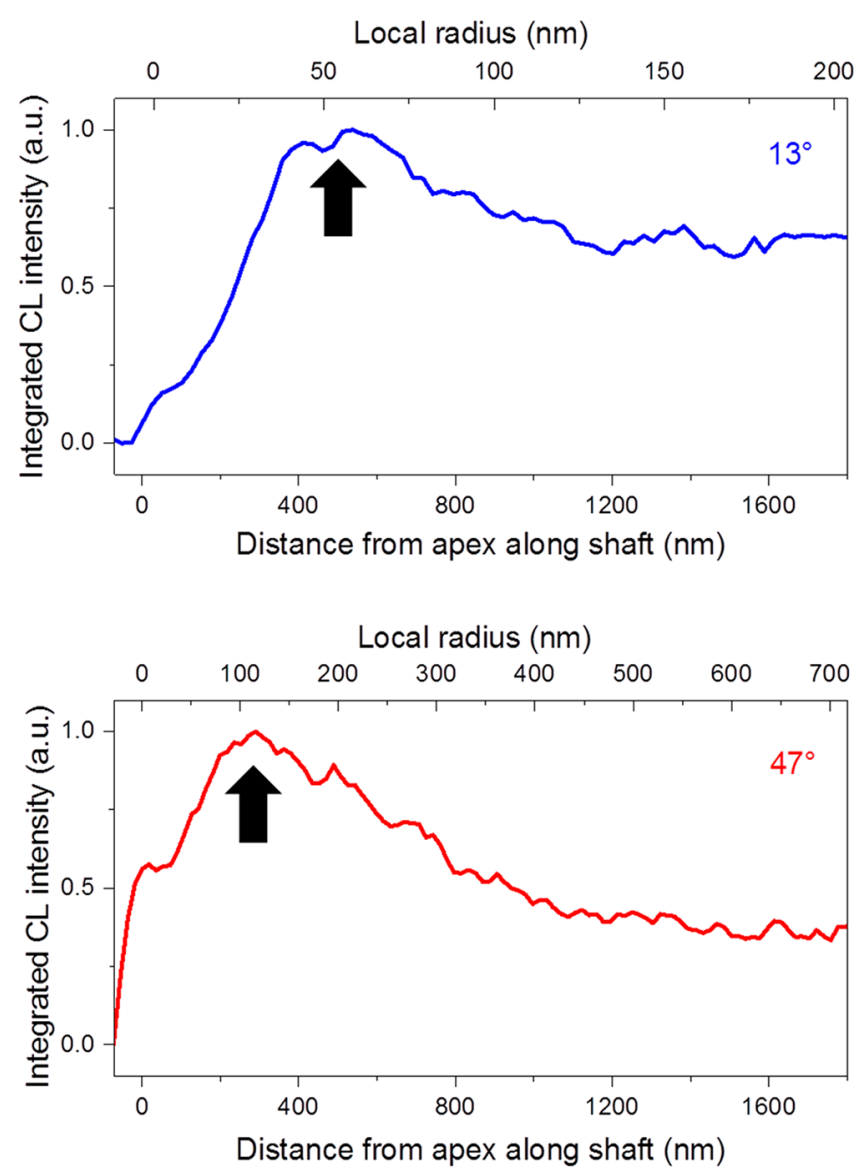

Figure 4. CL intensity integrated from 540 to $900 \mathrm{~nm}$ for tapers with $13^{\circ}$ (blue) and $47^{\circ}$ (red) opening angles plotted as a function of the distance from the apex along the shafts and the local taper radius, respectively. The black arrows highlight the locations with the highest CL intensity along the taper shafts.

decay of the $m=1$ mode. Since the $m=1$ mode is transformed from a bound, evanescent mode into a radiative mode near a critical radius of $\sim 100 \mathrm{~nm}$, most of the radiation that is detected in our experiments is emitted at a finite distance from the apex. Complementary to the previous study, ${ }^{39}$ the data present experimental evidence for the existence of a critical radius at which the higher order modes of tapers contribute to the radiation, ${ }^{16}$ thanks to the high spatial resolution of the STEM-CL experiments and the localized fast electron excitation. Our analysis shows that the CL results are mainly sensitive to the radiative losses of the different angular momentum modes of the conical taper, implying that ohmic losses are too weak to significantly affect the results.

\section{CONCLUSIONS}

We experimentally studied the far-field radiation of threedimensional plasmonic gold tapers with opening angles of $13^{\circ}$ and $47^{\circ}$ in proximity of their apexes by means of CL. Fast electrons launched a wave packet of surface plasmons around the shafts, and the sequential radiation decay of the plasmons was detected. The far-field radiation spectra of both tapers demonstrated a significant radiation between 500 and $700 \mathrm{~nm}$ due to the decay of higher order plasmonic modes $(|m| \geq 1)$ when the electrons excited the area behind the taper apex. This feature is independent of the opening angle. In contrast, the radiation directly coming from the very proximity of taper 
apexes is different: the $13^{\circ}$ taper has a remarkably weaker radiation from the apex compared to the $47^{\circ}$ taper. Numerical calculations showed that, on the taper with the smaller opening angle of $13^{\circ}$, up to $75 \%$ of the electron-induced power was guided away from the apex along the shaft, rather than being coupled to the far-field radiation. For the $47^{\circ}$ taper, adiabatic nanofocusing (and hence its reciprocal picture) fails and consequently leads to stronger radiation of the plasmonic modes at the apex. In addition, numerical results were computed by solving Maxwell's equations. The good agreement with the experimental results implies that the observed radiation phenomena of plasmonic tapers can be well understood as coherent radiation of higher order surface plasmon modes of the tapers. These results enrich the understanding and applications of plasmonic tapers as nanoscale light sources or absorbers in the field of enhanced Raman spectroscopy, scanning near-field optical microscopy, and biomolecule diagnostics.

\section{METHODS}

Three-Dimensional Gold Tapers. Polycrystalline gold wires with a diameter of $125 \mu \mathrm{m}$ were first annealed at $800{ }^{\circ} \mathrm{C}$ for $8 \mathrm{~h}$ and then slowly cooled to room temperature. These annealed wires were then electrochemically etched in hydrochloric acid with voltage pulses applied.

The length of the taper is around $50 \mu \mathrm{m} .^{35}$ The gold tapers were mounted onto TEM copper rings with conductive epoxy with the tips freestanding at the center of the ring. Plasma cleaning was applied beforehand to remove any contaminants from the taper surface.

$\mathrm{CL}$ Measurements. The experiments were performed using a Vacuum Generator HB501 scanning transmission electron microscope equipped with a cold field emission electron gun. CL measurements were carried out with an Attolight Mönch system at an operation voltage of $100 \mathrm{kV}$. The electron probe had a current of around $1 \mathrm{nA}$ and a size of a few nanometers. The solid detection angle is $1.2 \pi$ sr. The pixel size was around $20 \times 20 \mathrm{~nm}$, and the dwell time per pixel was $50 \mathrm{~ms}$.

Data Processing. The wavelengths and intensities of the acquired CL spectra were calibrated, and intensity spikes were removed. Principle component analysis (PCA) was then applied to reduce the noise of the spectra in Figures 2-4. The analysis was performed with the open source Python library HyperSpy V0.8.1. ${ }^{40}$ The spectral response of the CL spectrometer at different wavelengths was sequentially corrected. Finally, the background noise was subtracted from the spectra by taking the reference from the vacuum area. The experimental CL spectra extracted along taper shafts were summed over a width of 3 pixels $(\sim 70 \mathrm{~nm})$ in order to enhance the signals.

FDTD Simulations. A charge broadening scheme, as described elsewhere, was introduced to mimic the electron probe. ${ }^{41}$ The whole simulation domain has been discretized by unit cells of $1.5 \mathrm{~nm}$ edge lengths. The electron trajectory has been fixed at $1.5 \mathrm{~nm}$ away from the surface of the taper. The total scattered electromagnetic fields were calculated by solving Maxwell's equations embedded with the experimentally measured dielectric functions of gold, fitted to a Drude term plus two critical point functions. ${ }^{42-44}$ The Poynting vectors at the six orthogonal planes normal to the $\pm x, \pm y$, and $\pm z$ axes were computed, respectively. CL spectra were calculated as an integral of the Poynting vectors along the $-x,+y,-y$, and $+z$ directions in the $x \leq 0$ domain. The power flow through the planes normal to the $\pm x, \pm y$, and $\pm z$ axes represents the electron-induced radiation, whereas the energy captured by the plane normal to the $-z$ direction denotes the guided power propagating away from the apex.

\section{ASSOCIATED CONTENT}

\section{S Supporting Information}

The Supporting Information is available free of charge on the ACS Publications website at DOI: 10.1021/acsphotonics.9b00838.

Comparison between the simulated CL spectra and the experimental EEL spectra of a $13^{\circ}$ taper (S1), simulated CL spectra of a $13^{\circ}$ taper without an apex (S2), momentum-resolved energy loss spectra of $m=0$ and $m$ $=1$ modes on infinite gold fibers with different radii (S3) (PDF)

\section{AUTHOR INFORMATION}

\section{Corresponding Authors}

*E-mail: s.guo@flkf.mpg.de.

*E-mail: n.talebi@fkf.mpg.de.

ORCID ${ }^{\circ}$

Surong Guo: 0000-0003-2876-155X

Nahid Talebi: 0000-0002-3861-1005

Christoph Lienau: 0000-0003-3854-5025

Mathieu Kociak: 0000-0001-8858-0449

\section{Present Addresses}

${ }^{\perp}$ (A.C.) Facultad de Ciencias y Tecnología, Universidad Tecnológica de Panamá, Panamá.

"(M.E.) Centre for Nanoscience and Nanotechnology (C2N), CNRS, Université Paris-Sud, Université Paris-Saclay, 91120 Palaiseau, France.

\section{Author Contributions}

${ }^{\#}$ S. Guo and N. Talebi have equally contributed to this work. Notes

The authors declare no competing financial interest.

Portions of this work were presented in the Ph.D. thesis of S.G. (Section 3.4) published online in 2018, https://tuprints.ulb.tudarmstadt.de/7714/.

\section{ACKNOWLEDGMENTS}

N.T. acknowledges financial support from the European Research Council (ERC Starting Grant NanoBeam). This project has received funding from the European Union's Horizon 2020 Research and Innovation Programme under grant agreement no. 823717-ESTEEM3. C.L. acknowledges financial support by the Deutsche Forschungsgemeinschaft (SPP 1839 and SPP 1840). M.E. acknowledges funding by the Deutsche Forschungsgemeinschaft (401390650).

\section{REFERENCES}

(1) Babadjanyan, A. J.; Margaryan, N. L.; Nerkararyan, K. V. Superfocusing of surface polaritons in the conical structure. J. Appl. Phys. 2000, 87, 3785-3788.

(2) Stockman, M. Nanofocusing of Optical Energy in Tapered Plasmonic Waveguides. Phys. Rev. Lett. 2004, 93, 137404.

(3) Berweger, S.; Atkin, J. M.; Olmon, R. L.; Raschke, M. B. Light on the Tip of a Needle: Plasmonic Nanofocusing for Spectroscopy on the Nanoscale. J. Phys. Chem. Lett. 2012, 3, 945-952. 
(4) Li, Z.-Y. Mesoscopic and Microscopic Strategies for Engineering Plasmon-Enhanced Raman Scattering. Adv. Opt. Mater. 2018, 6, 1701097.

(5) Ma, X.; Zhu, Y.; Yu, N.; Kim, S.; Liu, Q.; Apontti, L.; Xu, D.; Yan, R.; Liu, M. Toward High-Contrast Atomic Force MicroscopyTip-Enhanced Raman Spectroscopy Imaging: Nanoantenna-Mediated Remote-Excitation on Sharp-Tip Silver Nanowire Probes. Nano Lett. 2019, 19, 100-107.

(6) Ropers, C.; Solli, D. R.; Schulz, C. P.; Lienau, C.; Elsaesser, T. Localized Multiphoton Emission of Femtosecond Electron Pulses from Metal Nanotips. Phys. Rev. Lett. 2007, 98, 043907.

(7) Li, S.; Jones, R. R. High-energy electron emission from metallic nano-tips driven by intense single-cycle terahertz pulses. Nat. Commun. 2016, 7, 13405.

(8) Vogelsang, J.; Talebi, N.; Hergert, G.; Wöste, A.; Groß, P.; Hartschuh, A.; Lienau, C. Plasmonic-Nanofocusing-Based Electron Holography. ACS Photonics 2018, 5, 3584-3593.

(9) Vogelsang, J.; Hergert, G.; Wang, D.; Groß, P.; Lienau, C. Observing charge separation in nanoantennas via ultrafast pointprojection electron microscopy. Light: Sci. Appl. 2018, 7, 55.

(10) Krüger, M.; Lemell, C.; Wachter, G.; Burgdörfer, J.; Hommelhoff, P. Attosecond physics phenomena at nanometric tips. J. Phys. B: At., Mol. Opt. Phys. 2018, 51, 172001.

(11) Bach, N.; Domröse, T.; Feist, A.; Rittmann, T.; Strauch, S.; Ropers, C.; Schäfer, S. Coulomb interactions in high-coherence femtosecond electron pulses from tip emitters. Struct. Dyn. 2019, 6, 014301.

(12) Müller, M.; Paarmann, A.; Ernstorfer, R. Femtosecond electrons probing currents and atomic structure in nanomaterials. Nat. Commun. 2014, 5, 5292.

(13) Talebi, N.; Sigle, W.; Vogelgesang, R.; Esmann, M.; Becker, S. F.; Lienau, C.; van Aken, P. A. Excitation of Mesoscopic Plasmonic Tapers by Relativistic Electrons: Phase Matching versus Eigenmode Resonances. ACS Nano 2015, 9, 7641-7648.

(14) Yalunin, S. V.; Schröder, B.; Ropers, C. Theory of electron energy loss near plasmonic wires, nanorods, and cones. Phys. Rev. B: Condens. Matter Mater. Phys. 2016, 93, 115408.

(15) Vogel, M. W.; Gramotnev, D. K. Optimization of plasmon nano-focusing in tapered metal rods. J. Nanophotonics 2008, 2, 021852 .

(16) Esmann, M.; Becker, S. F.; da Cunha, B. B.; Brauer, J. H.; Vogelgesang, R.; Gross, P.; Lienau, C. k-space imaging of the eigenmodes of sharp gold tapers for scanning near-field optical microscopy. Beilstein J. Nanotechnol. 2013, 4, 603-610.

(17) Neacsu, C. C.; Berweger, S.; Olmon, R. L.; Saraf, L. V.; Ropers, C.; Raschke, M. B. Near-Field Localization in Plasmonic Superfocusing: A Nanoemitter on a Tip. Nano Lett. 2010, 10, 592-596.

(18) Lu, F.; Zhang, W.; Huang, L.; Liang, S.; Mao, D.; Gao, F.; Mei, T.; Zhao, J. Mode evolution and nanofocusing of grating-coupled surface plasmon polaritons on metallic tip. Opto-Electronic Adv. 2018, 1,180010 .

(19) Schröder, B.; Weber, T.; Yalunin, S. V.; Kiel, T.; Matyssek, C.; Sivis, M.; Schäfer, S.; von Cube, F.; Irsen, S.; Busch, K.; Ropers, C.; Linden, S. Real-space imaging of nanotip plasmons using electron energy loss spectroscopy. Phys. Rev. B: Condens. Matter Mater. Phys. 2015, 92, 085411.

(20) Stockman, M. I.; Kneipp, K.; Bozhevolnyi, S. I.; Saha, S.; Dutta, A.; Ndukaife, J.; Kinsey, N.; Reddy, H.; Guler, U.; Shalaev, V. M.; Boltasseva, A.; Gholipour, B.; Krishnamoorthy, H. N. S.; MacDonald, K. F.; Soci, C.; Zheludev, N. I.; Savinov, V.; Singh, R.; Groß, P.; Lienau, C.; Vadai, M.; Solomon, M. L.; Barton, D. R.; Lawrence, M.; Dionne, J. A.; Boriskina, S. V.; Esteban, R.; Aizpurua, J.; Zhang, X.; Yang, S.; Wang, D.; Wang, W.; Odom, T. W.; Accanto, N.; de Roque, P. M.; Hancu, I. M.; Piatkowski, L.; van Hulst, N. F.; Kling, M. F. Roadmap on plasmonics. J. Opt. 2018, 20, 043001.

(21) Ropers, C.; Elsaesser, T.; Cerullo, G.; Zavelani-Rossi, M.; Lienau, C. Ultrafast optical excitations of metallic nanostructures: from light confinement to a novel electron source. New J. Phys. 2007, 9, 397.
(22) Thomas, S.; Wachter, G.; Lemell, C.; Burgdörfer, J.; Hommelhoff, P. Large optical field enhancement for nanotips with large opening angles. New J. Phys. 2015, 17, 063010.

(23) García de Abajo, F. J. Optical excitations in electron microscopy. Rev. Mod. Phys. 2010, 82, 209-275.

(24) Kociak, M.; Gloter, A.; Stéphan, O. A spectromicroscope for nanophysics. Ultramicroscopy 2017, 180, 81-92.

(25) Cherqui, C.; Thakkar, N.; Li, G.; Camden, J. P.; Masiello, D. J. Characterizing Localized Surface Plasmons Using Electron EnergyLoss Spectroscopy. Annu. Rev. Phys. Chem. 2016, 67, 331-357.

(26) Kociak, M.; Stéphan, O. Mapping plasmons at the nanometer scale in an electron microscope. Chem. Soc. Rev. 2014, 43, 38653883.

(27) Talebi, N. A directional, ultrafast and integrated few-photon source utilizing the interaction of electron beams and plasmonic nanoantennas. New J. Phys. 2014, 16, 053021.

(28) Losquin, A.; Kociak, M. Link between Cathodoluminescence and Electron Energy Loss Spectroscopy and the Radiative and Full Electromagnetic Local Density of States. ACS Photonics 2015, 2, 1619-1627.

(29) Talebi, N. Interaction of electron beams with optical nanostructures and metamaterials: from coherent photon sources towards shaping the wave function. J. Opt. 2017, 19, 103001.

(30) Losquin, A.; Zagonel, L. F.; Myroshnychenko, V.; RodríguezGonzález, B.; Tencé, M.; Scarabelli, L.; Förstner, J.; Liz-Marzán, L. M.; García de Abajo, F. J.; Stéphan, O.; Kociak, M. Unveiling Nanometer Scale Extinction and Scattering Phenomena through Combined Electron Energy Loss Spectroscopy and Cathodoluminescence Measurements. Nano Lett. 2015, 15, 1229-1237.

(31) Kawasaki, N.; Meuret, S.; Weil, R.; Lourenço-Martins, H.; Stéphan, O.; Kociak, M. Extinction and Scattering Properties of HighOrder Surface Plasmon Modes in Silver Nanoparticles Probed by Combined Spatially Resolved Electron Energy Loss Spectroscopy and Cathodoluminescence. ACS Photonics 2016, 3, 1654-1661.

(32) Tararan, A.; di Sabatino, S.; Gatti, M.; Taniguchi, T.; Watanabe, K.; Reining, L.; Tizei, L. H. G.; Kociak, M.; Zobelli, A. Optical gap and optically active intragap defects in cubic BN. Phys. Rev. B: Condens. Matter Mater. Phys. 2018, 98, 094106.

(33) Guo, S.; Talebi, N.; Campos, A.; Kociak, M.; van Aken, P. A. Radiation of Dynamic Toroidal Moments. ACS Photonics 2019, 6, $467-474$.

(34) Guo, S.; Talebi, N.; Sigle, W.; Vogelgesang, R.; Richter, G.; Esmann, M.; Becker, S. F.; Lienau, C.; van Aken, P. A. Reflection and Phase Matching in Plasmonic Gold Tapers. Nano Lett. 2016, 16, $6137-6144$

(35) Schmidt, S.; Piglosiewicz, B.; Sadiq, D.; Shirdel, J.; Lee, J. S.; Vasa, P.; Park, N.; Kim, D.-S.; Lienau, C. Adiabatic Nanofocusing on Ultrasmooth Single-Crystalline Gold Tapers Creates a 10-nm-Sized Light Source with Few-Cycle Time Resolution. ACS Nano 2012, 6, 6040-6048.

(36) Sánchez, E. J.; Novotny, L.; Xie, X. S. Near-Field Fluorescence Microscopy Based on Two-Photon Excitation with Metal Tips. Phys. Rev. Lett. 1999, 82, 4014-4017.

(37) Piglosiewicz, B.; Schmidt, S.; Park, D. J.; Vogelsang, J.; Groß, P.; Manzoni, C.; Farinello, P.; Cerullo, G.; Lienau, C. Carrierenvelope phase effects on the strong-field photoemission of electrons from metallic nanostructures. Nat. Photonics 2014, 8, 37.

(38) Potylitsyn, A. P.; Ryazanov, M. I.; Strikhanov, M. N.; Tishchenko, A. A. Diffraction Radiation from Relativistic Particles; Springer-Verlag: Berlin, Heidelberg, 2011.

(39) Ropers, C.; Neacsu, C. C.; Elsaesser, T.; Albrecht, M.; Raschke, M. B.; Lienau, C. Grating-Coupling of Surface Plasmons onto Metallic Tips: A Nanoconfined Light Source. Nano Lett. 2007, 7, 2784-2788.

(40) Peña, F. d. 1.; Burdet, P.; Ostasevicius, T.; Sarahan, M.; Magnunor, V.; Taillon, J.; Eljarrat, A.; Mazzucco, S.; Donval, G.; Zagonel, L. F.; Walls, M. iygr hyperspy: HyperSpy 0.8.1. DOI: $10.5281 /$ zenodo.27735. 
(41) Talebi, N.; Sigle, W.; Vogelgesang, R.; van Aken, P. Numerical simulations of interference effects in photon-assisted electron energyloss spectroscopy. New J. Phys. 2013, 15, 053013.

(42) Johnson, P. B.; Christy, R. W. Optical Constants of the Noble Metals. Phys. Rev. B 1972, 6, 4370-4379.

(43) Etchegoin, P. G.; Le Ru, E. C.; Meyer, M. An analytic model for the optical properties of gold. J. Chem. Phys. 2006, 125, 164705.

(44) Etchegoin, P. G.; Le Ru, E. C.; Meyer, M. An analytic model for the optical properties of gold. J. Chem. Phys. 2006, 125, 164705; J. Chem. Phys. 2007, 127, 189901. 